\title{
Theory of polymer gels with liquid crystal solvents
}

\author{
F. Brochard \\ Collège de France, 75231 Paris Cedex 05, France
}

(Reçu le 28 mars 1979, accepté le 17 juillet 1979)

\begin{abstract}
Résumé. - On étudie des filets de chaînes flexibles (ayant $N$ monomères entre points d'attache) gonflés dans un solvant nématogène (qui peut devenir nématique au-dessous d'une température $T_{\mathrm{c}}$ ).

1) Au-dessus de $T_{\mathrm{c}}$ une contrainte unidirectionnelle $t$ appliquée au gel induit un ordre nématique à l'intérieur du solvant. Ceci conduit à une large augmentation $\Delta n$ de la biréfringence. Le rapport $\Delta n / t$ est indépendant de $N$, en accord avec les mesures mécano-optiques de Gebhard et Rehage [1]. On prévoit une forte augmentation de $\Delta n / t$ au voisinage de $T_{\mathrm{c}_{0}}$.

2) Au-dessous de $T_{\mathrm{c}}$, on prévoit que le gel collapse quelle que soit la valeur de $N:$ la fraction $\varphi$ de monomères est très grande $(\varphi \gtrsim 0,1)$ et indépendante de $N$. A l'intérieur du gel collapsé, la température de la transition N-I est fortement réduite : l'état nématique disparaît souvent complètement. Cependant, un ordre nématique peut être maintenu dans un gel gonflé : $a$ ) par un refroidissement rapide d'un gel dilué ( $N$ grand); $b$ ) si les chaînes sont elles-mêmes nématogènes.
\end{abstract}

\begin{abstract}
We consider networks made of flexible chains (having $N$ monomers between crosslinks) swollen in a nematogenic solvent (a solvent which may become nematic below a certain temperature $T_{\mathrm{c}}$ ).

1) Above $T_{\mathrm{c}}$ a uniaxial stress $t$, applied to the network, induces a nematic order $S_{\mathrm{i}}$ inside the solvent. This leads to a large increase $\Delta n$ of the orientational birefringence. The ratio $\Delta n / t$ is independent of $N$, in agreement with stress-optical measurements performed by Gebhard and Rehage [1]. $\Delta n / t$ is predicted to increase as $T$ approaches $T_{\text {co }}$.

2) Below $T_{\mathrm{c}}$, we predict that the gel collapses into a dense state for all values of $N$. In this state, the volume fraction $\varphi$ of monomers is very large $(\varphi \gtrsim 0.1)$ and is independent of $N$. Inside the collapsed gel, the N-I transition temperature is strongly reduced : the nematic phase should often disappear completely. On the other hand, a swollen gel with a nematic solvent could be maintained : $a$ ) by suddenly cooling a dilute network ( $N$ large); $b$ ) is the polymer chains themselves are also nematogenic.
\end{abstract}

1. Introduction. - The static and dynamical properties of gels swollen in isotropic solvents have been intensively studied $[2,4,5,7,11]$. These properties have numerous industrial applications (foods, cosmetics, biomaterials...). The Flory theory of gels [2] gives a good account of their swelling in solvents of variable quality. Recent progress on the understanding of polymer solution, based on scaling laws [12], is also applicable to gels. A network of flexible chains (degree of polymerization $N$ ) is similar to a solution of free chains with the same $N$ value at the crossover concentration $c^{*}$ between the dilute and the semidilute regime [3]. This has been confirmed by neutron experiments probing the local conformation of the chains [4] and by inelastic light scattering for the dynamical properties [5]. For the particular problem of swollen gels, the predictions based on scaling or on a suitable form of the mean field (Flory) theory, are not very different.
Our aim in the present paper is to extend the Flory theory to networks swollen by mesomorphic solvents. Clearly a gel swollen by a nematic - or nearly nematic - liquid should show unusual mechanical and optical effects. In particular a large increase of the orientational birefringence has been observed with TPR (trans 1-5-polypentenamer) swollen in MBBA (N-p-methoxy benzylidene-p-butylaniline) above the nematic-isotropic transition temperature $T_{\mathrm{c}_{0}}$ of pure MBBA [1]. We study here the swelling of the gel at equilibrium both below and above the nematic/ isotropic transition point $T_{\mathrm{c}}$. We also discuss the orientation of the nematic molecules induced by a stretching of the network.

2. Swelling in conventional solvents. -2.1 EQUILIBRIUM DEGREE OF SWELLING. - A gel immersed in a good solvent swells because osmotic effects attract the solvent inside the gel. An elastic restoring pressure 
of the network opposes the swelling. The equilibrium equation $\mu_{\mathrm{s}_{\mathrm{i}}}=\mu_{\mathrm{s}_{\mathrm{e}}}$ between the chemical potential of the solvent inside the gel $\left(\mu_{\mathrm{s}_{\mathrm{i}}}\right)$ and outside $\left(\mu_{\mathrm{s}_{\mathrm{e}}}\right)$ expresses the balance between the osmotic and the elastic pressure.

Using a lattice model for the chains, Flory writes the free energy per site of the swollen gel in the form [2], [3] $\left(^{*}\right)$

$$
\begin{aligned}
\frac{F_{\text {site }}}{T}=(1-\varphi) \log (1 & -\varphi)+\chi_{0} \varphi(1-\varphi)+ \\
& +3 f \frac{\varphi}{N} \frac{R^{2}}{R_{0}^{2}}+\mu_{0}(1-\varphi)
\end{aligned}
$$

$\chi_{0}$ is a dimensionless quantity which characterizes the interaction energy per solvent molecule devided by $T$. $\varphi=c a^{3}$ is the volume fraction of monomers, $c$ is the number of monomers per $\mathrm{cm}^{3}, N$ is the number of statistical segments of the chains between crosslinks, $f$ is a constant which depends upon the functionality of the gel, $\mu_{0}$ is the chemical potentiel of the pure solvent, $R$ is the radius of gyration of the chain and is related to $\varphi$ by the packing condition

$$
\varphi \frac{R^{3}}{a^{3}}=N \delta
$$

$\delta$, as $f$, depends upon the functionality of the gel. $R_{0}$ is the radius of gyration of the ideal chain $\left(R_{0}^{2}=N a^{2}\right)$. The first term of eq. (1) represents the translational entropy of the solvent, the second term measures the interaction between solvent and monomers, the third term is the elastic energy for ideal chain extension (we have simplified this term, assuming a significant swelling, i.e. $R^{2} \gg N a^{2}$ ).

The chemical potential of the solvent is given by the derivative of the total free energy $F$ with respect to the number $n_{1}$ of solvent molecules, the number $n_{2}$ of solute monomers being fixed.

with

$$
\mu_{\mathrm{s}}=\frac{\partial}{\partial n_{1}}\left(n_{1}+n_{2}\right) F_{\mathrm{site}}(\varphi),
$$

$$
\varphi=\frac{n_{2}}{n_{1}+n_{2}} .
$$

This leads to :

$$
\mu_{\mathrm{s}_{\mathrm{i}}}=-\varphi^{2} \frac{\partial}{\partial \varphi}\left[F_{\mathrm{site}} / \varphi\right]
$$

The degree of swelling $\varphi^{-1}$ is derived from the equality of $\mu_{\mathrm{s}}$ in the swollen gel $\left(\mu_{\mathrm{s}_{\mathrm{i}}}\right)$ and in the solution $\left(\mu_{\mathrm{s}_{\mathrm{e}}}=\mu_{0}\right)$, i.e.

$$
\frac{1}{2} \frac{v_{0}}{a^{3}} \varphi^{2}-\frac{2}{3} f \frac{a^{2}}{R_{0}^{2}}\left(\frac{\varphi}{N}\right)^{1 / 3}=0
$$

where $v_{0} / a^{3}=1-2 \chi_{0}$ is the excluded volume bet-

$\left.{ }^{*}\right)$ We choose temperature units where the Boltzmann constant is equal to unity. ween monomers. Eq. (4) gives the equilibrium concentration of the gel

$$
\varphi=a^{1 / 5} v_{0}^{-3 / 5} N^{-4 / 5}\left(\frac{4}{3} f\right)^{3 / 5} \simeq c^{*} a^{3}
$$

giving

$$
R \sim R_{F}=N^{3 / 5} a .
$$

Each chain in the gel has a size comparable to the size $\left(R_{F}\right)$ of a single chain in the same solvent.

The swollen network adjusts its concentration at the value $c^{*}=N / R_{F}^{3}$, in agreement with the theorem quoted in section 1 .

2.2 ElONGATION OF THE NETWORK ; ORIENTATIONAL BIREFRINGENCE. - We apply a uniaxial stress in the $z$ direction. The network is elongated in the $z$ direction $\left(\lambda=L_{\|} / L_{\|_{0}}\right)$ and reduced in the perpendicular direction. Assuming a constant volume $\left(L_{\perp} / I_{\perp_{0}}=1 / \sqrt{\lambda}\right)$. The configuration of the chains between crosslinks is now characterized by two lengths, $R_{\|}$in the $z$ direction and $R_{\perp}$ in the $x y$ plane. Assuming an affine deformation of the gel, even at the scale of one individual chain, we set :

$$
\begin{aligned}
& R_{\|}=\lambda R \\
& R_{\perp}=\frac{1}{\sqrt{\lambda}} R
\end{aligned}
$$

where $R$ is related to $\varphi$ through eq. (2).

The free energy per site, including the stretching, is for a small deformation $(\lambda-1 \ll 1)$ :

$$
\begin{aligned}
& \frac{F_{\text {site }}}{T}=(1-\varphi) \log (1-\varphi)+\chi \varphi(1-\varphi)+ \\
& +f \frac{\varphi}{N}\left(\lambda^{2}+\frac{2}{\lambda}\right) \frac{R^{2}}{R_{0}^{2}}-\frac{t a^{3}}{T}(\lambda-1)+\mu_{0}(1-\varphi) .
\end{aligned}
$$

We have now two independent variables $\lambda$ and $\varphi$. They are derived from the minimization equations :

$$
\left\{\begin{array}{l}
\frac{\partial F_{\text {site }}}{\partial \lambda}=0 \\
\mu_{\mathrm{s}}=\mu_{0} .
\end{array}\right.
$$

It is convenient to introduce a parameter

$$
K=\frac{1}{N}\left(\frac{\varphi}{N}\right)^{1 / 3}
$$

In term of $K$, eq. (8) leads to

$$
e=\lambda-1=\frac{t}{E}
$$

where $E=6 f K T / a^{3}$ is an elongational elastic modulus of the gel, and to

$$
\frac{\delta \varphi}{\varphi}=-\frac{1}{30}\left(\frac{t a^{3}}{K}\right)^{2}
$$

showing that a stretching force increases the equilibrium swelling. The volume variation is of second order in the deformation $\left(\delta v / v \sim e^{2}\right)$ and our assumption of constant volume is well justified. 
Remark. - The Flory theory works because of the cancellation of two strong approximations contained in eq. (7) : $\alpha$ ) correlations between monomers are ignored. The full inclusion of correlation would lead to a smaller repulsive energy $\left(1 / 2 v_{0} c^{2} \rightarrow c^{2.25}\right.$ in eq. (4)) ; $\beta$ ) the non ideality of the chains leads to a smaller elastic energy of distortion

$$
\left(\frac{\varphi}{N} \frac{K_{\|}^{2}+2 R_{\perp}^{2}}{R_{F}^{2}} \text { instead of } \frac{\varphi}{N} \frac{R_{\|}^{2}+2 R^{2}}{R_{0}^{2}}\right) .
$$

Including these two corrections in eq. (4) maintains the $c^{*}$ theorem for $\varphi$.

Note however that the mean field eq. (7) does not lead to the correct gel modulus $E$. However including the above two corrections, we find :

$$
K=a^{3} / R^{3}=v a^{3}
$$

(where $v$ is the density of crosslinks) and $E=6 f v T$ is proportional to $v$ as expected. In all numerical applications, we will take for $K$ the corrected result (11).

Orientational birefringence. - The optical anisotropy coefficient of an ideal chain is known to be independent of $N$ and practically equal to the optical anisotropy of one monomer [6], [7]. The birefringence $\Delta n$ of a network of ideal chain caused by the relative deformation $\lambda$ is then

$$
\Delta n=b v \Delta \alpha\left(\frac{R_{\|}^{2}-R_{\perp}^{2}}{R_{\|}^{2}+2 R_{\perp}^{2}}\right)=b v \Delta \alpha(\lambda-1)
$$

where $\Delta \alpha=\alpha_{\|}-\alpha_{\perp}$ is the anisotropy of polarizability of one monomer and $b$ a constant independent of $N$.

It is convenient to form the ratio $C=\Delta n / t$. Using eqs. (9), (11) we have [1]

$$
C=\frac{b}{2 f} \frac{\alpha_{\|}-\alpha_{\perp}}{k T}
$$

$C$ is independent of $N$ and $T$ and is of the order of $10^{-9} \mathrm{~m}^{2} \mathrm{~N}^{-1}$.

3. Gels swollen in nematogenic solvents. - 3.1 PROPERTIES OF THE PURE SOLVENT [8]. - The gel is now immersed in an anisotropic solvent, which is nematic below a temperature $T_{\mathrm{c}_{0}}$. Below $T_{\mathrm{c}_{0}}$ the nematic order is characterized by a unit vector $\mathbf{n}$ (giving the direction of alignment of the molecules) and by a scalar $S$ (measuring the degree of molecular alignment along $\mathbf{n})$. Near $T_{\mathrm{c}_{0}}$ the chemical potential of the solvent may be expanded in power of $S$ as :

$$
\mu=\mu_{0}+\frac{1}{2} A_{0} S^{2}+\frac{1}{3} B S^{3}+\cdots
$$

with $A_{0}=\left(T-T_{\mathrm{c}_{0}}^{*}\right) d, d$ is a constant of order one. $\mu$ is not an even function of $S$ because the states $S$ and $(-S)$ are different. The isotropic to nematic transition is then always of first order and the transition temperature $T_{\mathrm{c}_{0}}$ is slightly larger than $T_{\mathrm{c}_{0}}^{*}$. In practice the nematic-isotropic transition is rather weakly first order [8] and $T_{\mathrm{c}_{0}}$ is very close to $T_{\mathrm{c}_{0}}^{*}$.

$$
\left[\frac{T_{\mathrm{c}_{0}}-T_{\mathrm{c}_{0}}^{*}}{T_{\mathrm{c}_{0}}} \simeq 2 \times 10^{-3}\right] \text {. }
$$

3.2 NETWORK IN ANISOTROPIC SOLVENT. - In an anisotropic matrix the configuration of the chains becomes anisotropic, as shown on figure 1 .

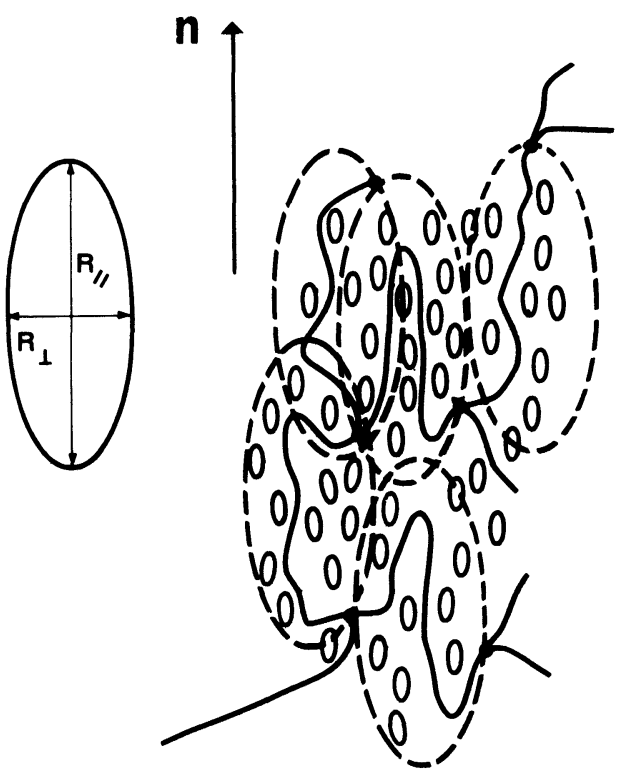

Fig. 1. - Gel swollen with a nematic solvent : polymer chains between crosslinks form ellipsoid coils (which can be prolate or oblate, depending upon nematic-monomer molecular interactions).

The general extension of the free energy per site (eq. (7)) including the spontaneous anisotropy of the gel induced by the nematogenic solvent may be written in the following way :

$$
\begin{aligned}
& \frac{F_{\text {site }}}{T}=(1-\varphi) \log (1-\varphi)+\tilde{\chi} \varphi(1-\varphi)+ \\
& \quad+f \frac{\varphi}{N}\left[\frac{R_{\|}^{2}}{R_{\|_{0}}^{2}}+\frac{2 R_{\perp}^{2}}{R_{\perp_{0}}^{2}}\right]+\mu(1-\varphi)-\frac{t}{T}(\lambda-1) a^{3}
\end{aligned}
$$

with $\tilde{\chi}=\chi_{0}+\chi_{1} S^{2}$

$$
\begin{aligned}
R_{\|_{0}}^{2}=R_{0}^{2}(1+k S), & R_{\perp_{0}}^{2}=R_{0}^{2}\left(1-\frac{k S}{2}\right) \\
R_{\|}=\lambda\left(\frac{N \delta}{\varphi}\right)^{1 / 3} a, & R_{\perp}=\frac{1}{\sqrt{\lambda}}\left(\frac{N \delta}{\varphi}\right)^{1 / 3} a .
\end{aligned}
$$


Here $\mu$ is given by eq. (14). For usual polymer nematic mixtures $\chi_{1}$ is positive and of order unity [10]. The excluded volume $v=a^{3}(1-2 \tilde{\chi})$ decreases as the nematic order appears and the quality of the solvent is lowered. It is however possible to expect $\chi_{1}$ to be small $\left(\chi_{1} \ll 1\right)$ if the monomers are also nematogenic.

Note that the spontaneous mean square length of the chain is now anisotropic $\left(R_{\|_{0}} \neq R_{\perp_{0}}\right)$. The expressions for $R_{\|}$and $R_{\perp}$ assure that the packing condition (eq. (2)) is satisfied $\left(R_{\|} R_{\perp}^{2} \varphi=N \delta a^{3}\right)$. The extension ratio $\lambda$ includes now both the spontaneous deformation of the gel and the elongation due to the stress $t$. The formulas for $R_{\|}$and $R_{\perp}$ ensure that 2 nd rank tensors are linear in $S$, while scalar terms contain contributions quadratic in $S$.

In the limit of small $\varphi$ and $S$, eq. (15) becomes

$$
\begin{aligned}
F_{\text {site }}=T\left[\frac{1}{2} \frac{v}{a^{3}} \varphi^{2}+3 K\right]+\frac{1}{2} A S^{2}-U S e a^{3}+ \\
+\frac{1}{2} E e^{2} a^{3}-t e a^{3}
\end{aligned}
$$

with $A=A_{0}(1-\varphi)+2 \chi_{1} \varphi \simeq\left(T-T_{\mathrm{c}}^{*}\right) d$,

$$
\left.\begin{array}{c}
\left(\frac{T_{\mathrm{c}}^{*}-T_{\mathrm{c}_{0}}^{*}}{T_{\mathrm{co}}^{*}}=\frac{-2 \chi_{1} \varphi}{d}\right) \\
\frac{v}{a^{3}}=1-2\left(\chi_{0}+\chi_{1} S^{2}\right) \\
U=3 k f K T / a^{3} \sim v T=\left(\varphi / N a^{3}\right) T \\
E=6 f K T / a^{3} \sim v T=\left(\varphi / N a^{3}\right) T
\end{array}\right\} .
$$

Eq. (16) leads to a reduction of the transition temperature inside the gel. If we ignore the difference between $T_{\mathrm{c}}^{*}$ and $T_{\mathrm{c}}$, this is given simply by

$$
\frac{T_{\mathrm{c}}-T_{\mathrm{c}_{0}}}{T_{\mathrm{c}_{0}}}=-2 \chi_{1} \varphi / d .
$$

We have to calculate the equilibrium values of $\varphi, S, e$ as a function of the temperature $T$ (or $S_{0}$, the order parameter of the solvent surrounding the gel) and of the applied stress $t$.

The equilibrium conditions are the following :

A) $\mu_{\mathrm{s}}$ is the same in and out of the gel :

$$
-\varphi^{2} \frac{\partial F_{\text {site }}}{\partial \varphi} / \varphi=\mu_{0}+\frac{1}{2} A_{0} S_{0}^{2}+O\left(S_{0}^{2}\right) .
$$

B) $F_{\text {site }}$ is minimal with respect to $S$ (the order parameter inside the gel)

$$
\frac{\partial F_{\text {site }}}{\partial S}=\frac{A}{a^{3}} S-U e+O\left(S^{2}\right)=0 .
$$
$\lambda$ :

C) $F_{\text {site }}$ is minimal with respect to the deformation

$$
\frac{\partial F_{\text {site }}}{\partial \lambda}=-U S+E e-t=0 .
$$

We shall solve these coupled equations in two cases :

a) Above $T_{\mathrm{c}_{0}}$ and under a uniaxial stress $t\left(S_{0}=0\right.$, $t \neq 0)$; a nematic order $S$ may be induced inside the gel $(S \neq 0)$ by the elongational stress;

b) Just below $T_{\mathrm{c}_{0}}\left(T_{\mathrm{c}}<T<T_{\mathrm{c}_{0}}\right)$. In this regime $S_{0} \neq 0$ but $S=0$. Here our main prediction is that the gel collapses (for finite, positive, $\chi_{1}$ ).

4. Behaviour above $\boldsymbol{T}_{\mathrm{c}_{0}}$. -4.1 NeMATIC ORDER INDUCED BY GEL ELONGATION. - At temperatures $T>T_{\text {co }}$, the nematogenic solvent is in its isotropic phase $\left(S_{0}=0\right)$ and the degree of swelling is given by eq. (5). An interesting new effect appears when a stress is applied to the gel. Because of the coupling between the elongation of the gel and the nematic alignment, a nematic order is induced inside the deformed gel. From eqs. (17), (18), (19), we get :

$$
S=\frac{U a^{3}}{A} e \sim \frac{\varphi}{N} \frac{T}{\Delta T} e
$$

with

$$
e=\frac{t}{6 f v T\left(1-\frac{U^{2}}{A E}\right)} \simeq \frac{t}{6 f v T} .
$$

Taking $\varphi \sim 10^{-2}, N \sim 100, T / \Delta T \sim 10^{2}$ we find $S \sim 0.01$ for $e \simeq 1$.

4.2 Orientational BiRefringence. - We have now two contributions to the birefringence :

- the direct contribution of the monomers given by eq. (12),

- the contribution of the solvent, proportional to $(1-\varphi) S$.

Then, we may write :

$$
\Delta n=\alpha S(1-\varphi)+3 b v\left(\alpha_{\|}-\alpha_{\perp}\right) e .
$$

For MBBA, we estimate $\alpha \sim 5$.

Inserting the result (20), we get (assuming $1-\varphi \ll 1)$ :

$$
\Delta n=3 b v\left(\alpha_{\|}-\alpha_{\perp}\right) e\left[1+\frac{k \alpha a^{3}}{2 C\left(T-T_{\mathrm{c}}^{*}\right)}\right]
$$

with $a=3 \AA, C=10^{-9}, \alpha=5$, and $\Delta T / T \sim 0.1$, this could lead to $\Delta n-\Delta n_{\text {iso }} / \Delta n_{\text {iso }} \sim 3$. With $\Delta T / T \sim 0.01$, we expect $\Delta n / \Delta n_{\text {iso }} \sim 30$ !

An increase of the birefringence has indeed been observed [1]. Contrary to the gel contribution (which is essentially temperature independent [9]) the nematic contribution should increase strongly as $T$ approaches $T_{\mathrm{c}}$. Because of the shift of $T_{\mathrm{c}}$ inside the gel, the smallest value of $T-T_{\mathrm{c}}^{*}$ is $T_{\mathrm{c}_{0}}-T_{\mathrm{c}_{0}}^{*}+2 \chi_{1} \varphi T_{\mathrm{c}_{0}} / d$. The largest accessible value of $\Delta n-\Delta n_{\text {iso }} / \Delta n_{\text {iso }}$ is then obtained in dilute gels $\left(\chi_{1} \varphi \rightarrow 0\right)$ and is given by :

$$
\left[\frac{\Delta n-\Delta n_{\text {iso }}}{\Delta n_{\text {iso }}}\right]_{\text {Max }}=\frac{k \alpha a^{3}}{2 C\left[T_{\mathrm{c}_{0}}-T_{\mathrm{c}_{0}}^{*}\right.} \text {. }
$$


For a concentrated gel, $T-T_{\mathrm{c}}^{*} / T$ cannot be small $\left(T-T_{\mathrm{c}}^{*} / T>\chi_{1} \varphi / d\right)$ and $\Delta n$ is reduced. Gebhard et al. [1] have obtained $\Delta n-\Delta n_{\text {iso }} / \Delta n_{\text {iso }} \simeq 3$ for $\varphi=0.5$.

5. Behaviour below $\boldsymbol{T}_{\mathrm{c}_{0}} \cdot-5.1$ EQUILIBRIUM DEGREE OF SWELLING. - Starting from a gel swollen by the nematic solvent above $T_{\mathrm{c}}$, we decrease the temperature smoothly. Just below $T_{\mathrm{co}}$, a nematic order appears in the pure solvent but the gel remains isotropic as long as $\left|T-T_{\mathrm{c}_{0}} / T_{\mathrm{c}_{\mathrm{o}}}\right|<2 \chi_{1} \varphi / d$ where $\varphi$ is the monomer concentration of the gel in equilibrium with the pure solvent. $\varphi$ is calculated from the condition $\mu_{\mathrm{s}}=$ constant. Eq. (17) with $S=0, S_{0} \neq 0$ leads to :

$$
\frac{1}{2} \frac{v_{0}}{a^{3}} \varphi^{2}-\frac{2}{3} f \frac{a^{2}}{R_{0}^{2}}\left(\frac{\varphi}{N}\right)^{1 / 3}=\frac{\mu-\mu_{0}}{T} .
$$

Just above $T_{\mathrm{c}}, \mu=\mu_{0}$ and $\varphi \simeq c^{*} a^{3} \sim 10^{-2}$ for $N=100$. The first term of equality (24) is then of the order of $10^{-4}$.

As soon as a nematic order appears in the pure solvent, the chemical potential of the solvent decreases outside the gel and some solvent inside the gel must be expelled. We can estimate the decrease of $\mu$ by :

$$
\frac{\mu-\mu_{0}}{T} \simeq \frac{1}{2} \frac{\left|T-T_{\mathrm{c}_{0}}^{*}\right|}{T} S_{0}^{2} \simeq 10^{-2} .
$$

A large osmotic pressure is needed in the gel to balance the appearance of the nematic order and the gel collapses. The second elastic term of eq. (24) which opposes gel extension is negligible here, and the equation for $\varphi$ is simply

$$
\frac{1}{2} \frac{v_{0}}{a^{3}} \varphi^{2}=\frac{1}{T}\left[\mu(T)-\mu_{0}\right]
$$

$\varphi$ is now independent of $N$. This result holds as long as $S=0$ inside the gel, that is for :

$$
\frac{\left|T-T_{\mathrm{co}_{0}}\right|}{T_{\mathrm{c}_{0}}}<2 \chi_{1} \varphi / d .
$$

The limiting concentration $\varphi_{1}$ such that

$$
\frac{\left|T-T_{\mathrm{c}_{0}}\right|}{T_{\mathrm{c}_{0}}}=2 \chi_{1} \varphi_{1} / d
$$

is derived from eq. (25). By taking

$$
\frac{1}{T}\left(\mu(T)-\mu_{0}\right)=\frac{1}{2}\left[\frac{2 \chi_{1} \varphi_{1}}{d}+\frac{T_{\mathrm{c}_{0}}-T_{\mathrm{c}_{0}}^{*}}{T_{\mathrm{c}_{0}}}\right] S_{0}^{2}
$$

we get :

$$
\frac{1}{2} \frac{v_{0}}{a_{3}} \varphi_{1}^{2}=\frac{1}{2}\left[\frac{2 \chi_{1} \varphi_{1}}{d}+\frac{T_{\mathrm{c}_{0}}-T_{\mathrm{c}_{0}}^{*}}{T_{\mathrm{c}_{0}}}\right] S_{0}^{2} .
$$

Taking $\chi_{1}$ of order unity, the first term is dominant and we get :

$$
\frac{1}{2} \frac{v_{0}}{a^{3}} \varphi_{1}=\chi_{1} S_{0}^{2} / d
$$

we find that $\varphi_{1}$ is very large $\left(\varphi_{1} \sim S_{0}^{2} \sim 0.5-0.1\right)$.

Thus the gel is too dense : for finite (positive) values of the parameter $\chi_{1}$, the nematic solvent cannot effectively penetrate much into the gel. This is in agreement with the observations of ref. [1] using MBBA as the solvent. In the future, it may become possible to find a system polymer-nematic with $\chi_{1}$ very small if the polymer is itself a nematogen. In this case, we expect a collapse of the gel in a certain interval $T_{\mathrm{c}}^{\prime}<T<T_{\mathrm{c}_{0}}$, where $\left|T_{\mathrm{c}}^{\prime}-T_{\mathrm{c}_{0}}\right| / T_{\mathrm{c}_{0}}=2 \chi_{1} \varphi_{1} / d$ with $\varphi_{1}$ defined by eq. (27). But below $T_{c}^{\prime}$, the gel should swell again! This may lead to interesting effects.

5.2 SUPERCOOLING OF THE Gel AT CONSTANT $\varphi$. We consider a loose gel ( $N$ large). If the temperature is suddenly decreased below $T_{\mathrm{c}}=T_{\mathrm{c}_{0}}\left[1-2 \chi_{1} \varphi / d\right] \varphi$ remains constant and a nematic order should appear inside the gel too. The order parameter $S$ in the gel is slightly smaller than $S_{0}$. The difference $S-S_{0}$ can be evaluated as :

$$
S-S_{0} \simeq \frac{\partial S}{\partial T}\left(T_{\mathrm{c}_{0}}-T_{\mathrm{c}}\right) \simeq \frac{2 \chi_{1} \varphi}{d}\left(T \frac{\partial S}{\partial T}\right) \sim 10^{-2} .
$$

It would be interesting to cool the gel in a magnetic field and to generate a nematic monodomain (with the nematic axis $\mathbf{n}$ pointing in the field direction). Magnetic fields of a few kilogauss are sufficient to align bulk nematic samples [8]. In our case alignment may be more difficult. But the onset of nematic order would lead to a macroscopic deformation of the gel. From eq. (19) valid for small deformation, we get :

$$
e=\lambda_{0}-1=\frac{k S}{2} .
$$

This is a strong effect. With $k \sim 1, S=0.7$, we expect $e \simeq 1 / 3$. A simple observation of the deformation of the gel could lead to a measurement of the parameter $k$ (which specifies the anisotropy of a polymer chain in an anisotropic matrix).

5.3 INSTABILITIES OF THE SUPERCOOLED GEL. Two situations may appear when we allow the supercooled gel to relax :

A) The gel remains stable and slowly expels the solvent.

B) A spinodal decomposition may be induced by a sudden cooling [11].

As $T$ is lowered at constant $\varphi, v$ decreases and fluctuations in concentration are enhanced. As $v$ reaches a critical value $v_{\mathrm{s}}$ [3] such that $\partial^{2} F / \partial \varphi^{2}=0$, 
microsyneresis takes place. We have here a competition between two instabilities : $a$ ) the spinodal decomposition, $b$ ) the nematic transition. The spinodal is somewhat similar to a second order phase transition, while the nematic phase occurs through a first order transition. We show on figure 2 a tentative phase diagram describing these features.

6. Concluding remarks. - We have found that gels swollen in nematogenic solvents should have remarkable mecano-optical properties :

1) Above $T_{\mathrm{c}}$, a deformation of the gel orients the nematic molecules. This could lead to enormeous increases in the birefringence ( $\Delta n$ anisotropic/ $\Delta n$ isotropic $\sim 30$ ) when $T$ approaches $T_{\mathrm{c}_{0}}$, at least in the case of dilute gels $\left(\varphi \sim 10^{-2}\right)$. Up to now the gels studied experimentally were only weakly swollen by nematogens such as MBBA $(\varphi \sim 0.5)$. In this case we expect a smaller effect $\left(\Delta n / \Delta n_{\text {iso }} \sim 3\right)$, which has been effectively observed by Gebhard et al.

2) Below $T_{c_{0}}$ we find that the gel should collapse whatever the initial degree of swelling. This has been observed with MBBA by a German group [1]. However starting from a dilute gel, one could obtain a gel swollen in a nematic matrix by a rapid cooling. By cooling the gel in a magnetic field, we expect that the macroscopic shape of the gel will change as the nematic order appears. Gels swollen by a nematic could also be obtained by using nematogenic polymers.

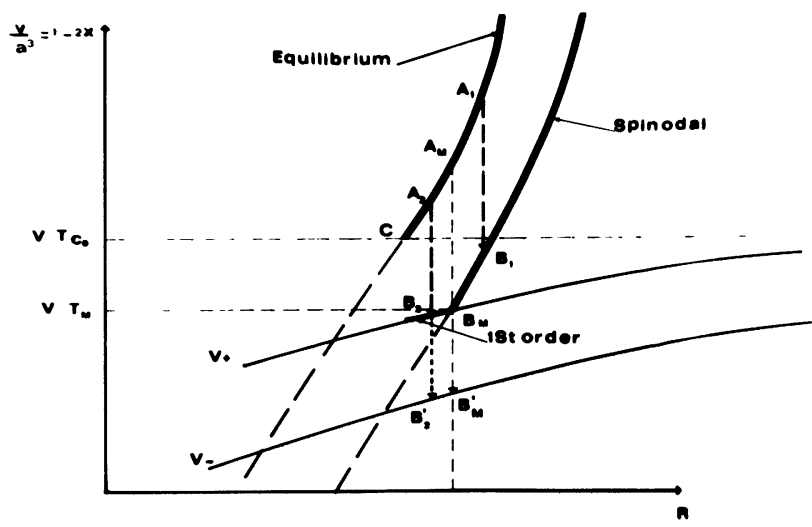

Fig. 2. - Phase diagram for a network swollen by a nematogen : the coordinates are the excluded volume parameter $v$ which varies in the same way as the temperature and the distance $R$ between crosslinks. The curve A represents the swollen gel in equilibrium with the solvent in its isotropic phase. At point $\mathrm{C}$, the gel collapses. The curve $v^{+}$represents the nematic-isotropic transition of the solvent inside the gel. The solvent is isotropic above $v^{\top}$ and nematic below. $v^{-}$is the excluded volume parameter just below $T_{\mathrm{c}}$. The curve $B$ is the spinodal : starting from a state $A$, a sudden cooling at constant $R$ is represented by a vertical line. Starting from $\mathrm{A}_{1}$, fluctuations in the monomer concentration increase and microsyneresis occurs at point $B_{1}$. Starting from $A_{2}$, we expect a sudden decomposition at point $\mathrm{B}_{2}$, due to the discontinuity of $v$ at the onset of nematic order.

Acknowledgments. - We are grateful to G. Rehage for calling our attention to this problem and to D. Cannell and P. G. de Gennes for helpful discussions.

\section{References}

[1] Gebhard, G., Rehage, G. and Schwarz, J., Non Crystalline Solids (1977) p. 81-87.

[2] FlORY, P., Principles of polymer chemistry (Cornell Univ. Press, Ithaca N. Y., 8th printing) 1971.

[3] De Gennes, P. G., Scaling concepts in Polymer Physics (Cornell Univ. Press) 1979.

[4] Benoit, H., DeCKeR, D., Duplessix, R., Picot, C., Rempr, P., Cotton, J. P., Farnoux, J. P., Jannink, G., Ober, R., J. Polym. Sci. A2 14 (1976) 2119.

[5] Munch, J. P., Candau, S., Duplessix, R., Picot, C. Benort, H., J. Physique Lett. 35 (1974) L-239.

Tanaka, T., Hocker, L., Benedeck, G., J. Chem. Phys. 59 (1973) 5151
[6] KuHN, W., Kolloid Z 68 (1934) 2.

Tsvetkov, V. N., Pokl. Akad. Nauk SSSR 165 (1965) 360.

[7] Treloar, L. R. G., The Physics of Rubber Elasticity (3rd edition Oxford) 1975

[8] De Gennes, P. G., The Physics of Liquid Crystals (Clarendon Press, Oxford) 1974.

[9] Gebhard, G., Thesis, Gera/Thüringen (1978).

[10] Kronberg, B., Bassignana, I., Patterson, D., J. Phys. Chem. 82 (1978) 1714.

[11] Tanaka, T., Ishiwata, S., Ischimoto, C., Phys. Rev. Lett. 58 (1977) 771.

[12] De Gennes, P. G., Nuovo Cimento 7 (1977) 363. 